\title{
Modified doublet lattice method for its analytical continuation in the complex plane
}

\author{
David Quero* \\ DLR, German Aerospace Center, 37073, Göttingen, Germany
}

$\begin{array}{ll} & \\ a_{k} & =\text { Nomenclature } \\ b_{n} & =\text { exponent coefficient in the series expansion } \\ \beta & =\sqrt{1-M_{\infty}^{2}} \\ \mathbf{D} & =\text { aerodynamic influence coefficient matrix } \\ \mathbf{D}_{1 j k}, \mathbf{D}_{1 j k} & =\text { substantial derivative matrices } \\ i & =\text { imaginary unit } \\ I_{1}, I_{2} & =\text { integrals related to the kernel function } \\ I_{0}, J_{0} & =\text { integrals to be approximated by the series expansion } \\ f, f_{N} & =\text { function and corresponding approximation with } N \text { terms } \\ g & =\text { damping, } g=\text { Re }(p) \\ k & =\text { reduced frequency, } k=\text { Im }(p) \\ p & =\text { nondimensional complex Laplace variable } \\ \mathbf{H}_{k g} & =\text { spline matrix } \\ M_{\infty} & =\text { Mach number } \\ R e & =\text { real part } \\ I m & =\text { imaginary part } \\ \mathbf{S}_{k j} & =\text { summation matrix } \\ \mathbf{Q}_{h h} & =\text { generalized aerodynamic forces matrix } \\ (x, y, z) & \text { coordinates of the receiving point } \\ (\xi, \eta, \zeta) & \text { coordinates of the sending point } \\ & \end{array}$

\section{Introduction}

$\mathrm{T}$ He subsonic doublet lattice method (DLM) has been a standard tool for aeroelastic analysis for several decades now [1]. Several aeroelastic applications based on the DLM for the description of the unsteady aerodynamics 
include the computation to gust responses and flutter stability analysis [2]. Despite the limitations associated to the potential flow to describe nonlinear effects present in the transonic regime, the DLM satisfactorily describes unsteady linearized aerodynamic phenomena in the subsonic regime. In this note the standard implementation of the DLM [1, 3] as extensively used for subsonic flow and implemented in MSC.Nastran [4, 5] is considered.

For a particular geometry and corresponding panel discretization, the standard DLM implementation was conceived for the computation of unsteady aerodynamic pressures as function of the Mach number $M_{\infty}$ and the reduced frequency $k$. The restriction of its validity to the imaginary axis causes in general a wrong damping (real part) prediction of the roots to the flutter equation when used in combination with the well-known $p-k$ method [6] or its modified version by Rodden et al. [4], as the complex value of the aerodynamic transfer function matrix is assumed to be equal to its value at the imaginary axis, which is in general not true. The $g$ method presented by Chen [7] introduced a first-order approximation for the analytical continuation of the aerodynamic transfer function matrix outside the imaginary axis, but remains limited to values of the complex variable close to the imaginary axis.

Edwards [8] showed that there is no actual restriction for the DLM to be applied solely to the imaginary axis. Edwards and coworkers derived aerodynamic transfer functions as dependent on the complex nondimensional Laplace variable $p$ including two-dimensional subsonic [8-[10] and supersonic [8, 10] speeds as well as three-dimensional subsonic speeds [11]. Edwards [11], Cunningham and Desmarais [12] and Hounjet and Eussen [13] generalized DLM computer codes for the computation of the aerodynamic transfer function with the Laplace variable $p$. This allows for a correct prediction of the roots to the flutter equation so that the use of approximate solutions such as the $p$ - $k$ or $g$ methods are not required, as the nonlinear eigenvalue problem can be readily solved upon dependence on the complex variable $p$. Edwards [11] did not explicitly provide the required modifications to the integrals $I_{0}$ and $J_{0}$ and restricted the applications to planar configurations. References [12, 13] provide explicit expressions but are likewise restricted to planar configurations, as they exclusively modify the integral term $I_{0}$ ignoring $J_{0}$, which does not contribute for planar geometries. For nonplanar configurations the latter integral $J_{0}$ is in general non-zero and its modification for general values of the complex variable $p$ is the main purpose of the current work. Thus, this note provides expressions modifying the standard DLM formulation for three-dimensional nonplanar configurations for its use with the complex Laplace variable $p$, extending its validity outside of the imaginary axis and enabling the use of the generalized aeroelastic analysis method (GAAM) as proposed by Edwards and Wieseman [14] for general configurations. After the presentation of the required modifications for the integral terms involved, results are shown for a planar and a nonplanar configuration. Note that the $p-k$ and $g$ methods are valid for both planar and nonplanar configurations, being their limitation related to the approximation applied outside the imaginary axis. 


\section{DLM formulation}

In this note the complete formulation of the DLM method is not explicitly provided, focusing on the terms relevant to enable its analytical continuation throughout the complex plane. There are several publications concerned with the implementation of the DLM, with further refinements improving the numerical approximations required. The formulation provided by Rodden et al. [3] considers a higher-order (quartic) approximation for the spanwise variation of the numerator of the incremental oscillatory kernel related to the doublet line segment which allows for a better description of the aerodynamic transfer function at higher frequencies and for higher panel aspect ratios, whilst the original formulation [1] used a parabolic approximation.

The computation of the integrals involving the subsonic kernel functions requires the evaluation of the integrals $I_{1}$ and $I_{2}[1,3,15]$ :

$$
\begin{aligned}
I_{1}\left(u_{1}, k_{1}\right) & =\int_{u_{1}}^{\infty} \frac{e^{-i k_{1} u}}{\left(1+u^{2}\right)^{3 / 2}} d u=e^{-i k_{1} u_{1}}\left[1-\frac{u_{1}}{\sqrt{1+u_{1}^{2}}}-i k_{1} I_{0}\left(u_{1}, k_{1}\right)\right], \\
I_{2}\left(u_{1}, k_{1}\right)=\int_{u_{1}}^{\infty} \frac{e^{-i k_{1} u}}{\left(1+u^{2}\right)^{5 / 2}} d u & =\frac{e^{-i k_{1} u_{1}}}{3}\left[\left(2+i k_{1} u_{1}\right)\left(1-\frac{u_{1}}{\sqrt{1+u_{1}^{2}}}\right)-\frac{u_{1}}{\left(1+u_{1}^{2}\right)^{3 / 2}}-i k_{1} I_{0}\left(u_{1}, k_{1}\right)+k_{1}^{2} J_{0}\left(u_{1}, k_{1}\right)\right]
\end{aligned}
$$

where:

$$
u_{1}=\frac{M_{\infty} \sqrt{(x-\xi)^{2}+\beta^{2}\left[(y-\eta)^{2}+(z-\zeta)^{2}\right]}-(x-\xi)}{\beta^{2} \sqrt{(y-\eta)^{2}+(z-\zeta)^{2}}}
$$

and $\beta=\sqrt{1-M_{\infty}^{2}}$. The coordinates of the receiving point are $(x, y, z)$ and those of the sending points, where the integration is carried out, $(\xi, \eta, \zeta)$. In Eqs. 1] and 2] and for the sake of harmonization with previous literature [1, 3, 15] the integration variable has been denoted by $u$, whereas the values of the integrals for each specific pair of the variable $u$ and reduced frequency are denoted by $\left(u_{1}, k_{1}\right)$. The additional integrals which appear in Eqs. 1 and $2\left(I_{0}\right.$ and $\left.J_{0}\right)$ are defined as:

$$
\begin{aligned}
& I_{0}\left(u_{1}, k_{1}\right)=e^{i k_{1} u_{1}} \int_{u_{1}}^{\infty}\left(1-\frac{u}{\sqrt{1+u^{2}}}\right) e^{-i k_{1} u} d u \\
& J_{0}\left(u_{1}, k_{1}\right)=e^{i k_{1} u_{1}} \int_{u_{1}}^{\infty} u\left(1-\frac{u}{\sqrt{1+u^{2}}}\right) e^{-i k_{1} u} d u .
\end{aligned}
$$

Note that due to the split of the subsonic kernel in planar and nonplanar terms [1], the contribution of the integral $I_{2}$ 
and consequently $J_{0}$ is not relevant for planar configurations, for which only the integral $I_{0}$ needs to be considered.

In the standard implementation of the DLM the following identities when computing the integrals $I_{1}$ and $I_{2}$ for the case $u_{1}<0$ are used [15]:

$$
\begin{aligned}
& I_{1}\left(u_{1}, k_{1}\right)=2 \operatorname{Re}\left\{I_{1}\left(0, k_{1}\right)\right\}-\operatorname{Re}\left\{I_{1}\left(-u_{1}, k_{1}\right)\right\}+i \operatorname{Im}\left\{I_{1}\left(-u_{1}, k_{1}\right)\right\}, \\
& I_{2}\left(u_{1}, k_{1}\right)=2 \operatorname{Re}\left\{I_{2}\left(0, k_{1}\right)\right\}-\operatorname{Re}\left\{I_{2}\left(-u_{1}, k_{1}\right)\right\}+i \operatorname{Im}\left\{I_{2}\left(-u_{1}, k_{1}\right)\right\},
\end{aligned}
$$

restricting the domain of the variable $u_{1}$ to $u_{1} \geq 0$ and thereby reducing the number of integral computations to be carried out. The use of these expressions is however valid only for the case where $k_{1}$ is a real number, $k_{1} \in \mathbb{R}$, and thus restricted to the imaginary axis in the complex plane $p$. For the computation of the $I_{1}$ and $I_{2}$ as function of the complex Laplace variable $p$, analytical continuation by means of the change of variables $k_{1}=p / i=\operatorname{Im}(p)-i \operatorname{Re}(p)$ is applied, resulting in a complex reduced frequency $k_{1} \in \mathbb{C}$. For a general complex value of the reduced frequency $k_{1}$ with an imaginary component, as required for the evaluation throughout the complex plane, the conjugate symmetry of the term $e^{-i k_{1} u}$ in the numerator of the integrands involved in the computation of $I_{1}$ and $I_{2}$ is lost. The author believes that the use of Eqs. 5 and 6 has restricted the use of the standard DLM to real values of the reduced frequency, constraining its validity to the imaginary axis in the complex plane defined by $p$. In the next section the modified expressions for the integrals $I_{1}$ and $I_{2}$ when $u_{1}<0$ for the general case $k_{1} \in \mathbb{C}$, which requires the computation of the integrals $I_{0}$ and $J_{0}$, are provided.

\section{GAAM for general nonplanar configurations}

The computation of the integrals $I_{0}$ and $J_{0}$ as provided in Eqs. 3 and 4 reduces to the evaluation of integrals involving the function $f(u)$ :

$$
f(u)=1-\frac{u}{\sqrt{1+u^{2}}} .
$$

Various approximations of this function have been used in order to analytically evaluate the integrals $I_{0}$ and $J_{0}$. They include the original Watkins approximation [16], the Laschka approximation [17] and several approximations due to Desmarais [18]. All of these approximations $f_{N}(u) \approx f(u)$ are given by a finite sum of exponential functions and can be written in the form:

$$
f_{N}(u)= \begin{cases}\sum_{n=1}^{N} a_{n} e^{-b_{n} u}, & u \geq 0 \\ 2-\sum_{n=1}^{N} a_{n} e^{-b_{n}|u|}, & u<0 .\end{cases}
$$


In particular the approximation D12.1 $(N=12)$ due to Desmarais is commonly used, where $b_{n}=2^{n / m} b$, with the corresponding coefficients provided in references [18] and [3]. In virtue of this exponential approximation the integrals $I_{0}$ and $J_{0}$ can be analytically carried out. For the case $u_{1} \geq 0$ the following expressions are obtained:

$$
\begin{gathered}
I_{0}\left(u_{1}, k_{1}\right)=\sum_{n=1}^{N} \frac{a_{n} e^{-b_{n} u_{1}}\left(b_{n}-i k_{1}\right)}{b_{n}^{2}+k_{1}^{2}}, u_{1} \geq 0, \\
J_{0}\left(u_{1}, k_{1}\right)=\sum_{n=1}^{N} \frac{a_{n} e^{-b_{n} u_{1}}\left(1+\left(b_{n}+i k_{1}\right) u_{1}\right)}{\left(b_{n}+i k_{1}\right)^{2}}, u_{1} \geq 0 .
\end{gathered}
$$

For the case of planar configurations Cunningham and Desmarais [12] and Hounjet and Eussen [13] explicitly provided modifications to integral $I_{0}$ (and thus $I_{1}$ ) for its analytical continuation in the complex variable $p$, which requires its computation for the case $u_{1}<0$ instead of using the expression given in Eq. 5 . In this note the corrected versions for the general nonplanar case involving both integrals $I_{0}$ and $J_{0}$ (and thus $I_{1}$ and $I_{2}$ ) when $u_{1}<0$ are derived by splitting the integration interval in the variable $u_{1}$ at $u=0$ and noting that $f(u)=2-f(|u|)$ for $u<0$. The resulting expressions are:

$$
\begin{gathered}
I_{0}\left(u_{1}, k_{1}\right)=\frac{2 i}{k_{1}}\left(e^{i k_{1} u_{1}}-1\right)-\sum_{n=1}^{N} \frac{a_{n}\left(e^{i k_{1} u_{1}}-e^{-b_{n} u_{1}}\right)\left(b_{n}-i k_{1}\right)}{b_{n}^{2}+k_{1}^{2}}+e^{i k_{1} u_{1}} I_{0}\left(0, k_{1}\right), u_{1}<0, \\
J_{0}\left(u_{1}, k_{1}\right)=\frac{2}{k_{1}^{2}}\left(e^{i k_{1} u_{1}}-i k_{1} u_{1}-1\right)+\sum_{n=1}^{N} \frac{a_{n}\left(e^{i k_{1} u_{1}}+e^{b_{n} u_{1}}\left(\left(b_{n}-i k_{1}\right) u_{1}-1\right)\right)}{\left(b_{n}-i k_{1}\right)^{2}}+e^{i k_{1} u_{1}} J_{0}\left(0, k_{1}\right), \quad u_{1}<0 .
\end{gathered}
$$

Once the corrected expressions for $I_{0}\left(u_{1}, k_{1}\right)$ and $J_{0}\left(u_{1}, k_{1}\right)$ for the general case of a complex reduced frequency $k_{1}=p / i=\operatorname{Im}(p)-i \operatorname{Re}(p) \in \mathbb{C}$ have been computed, the values for the integrals $I_{1}\left(u_{1}, k_{1}\right)$ and $I_{2}\left(u_{1}, k_{1}\right)$ can be obtained from Eqs. 1 and 2 in a straightforward manner, as they are valid for both $u_{1} \geq 0$ and $u_{1}<0$. Note that these modifications represent the analytical continuation of the original integrals $I_{1}\left(u_{1}, k_{1}\right)$ and $I_{2}\left(u_{1}, k_{1}\right)$, as they are not defined for values of the complex reduced frequency such that $\operatorname{Re}(p) \leq 0$, that is, $\operatorname{Im}\left(k_{1}\right) \geq 0$.

\section{Results}

In this section results obtained with the modified DLM valid for the analytical continuation throughout the complex plane $p$, with the terms $I_{0}$ and $J_{0}$ computed according to Eqs. 7, 8, 9, and 10, is validated against the results obtained by a first-order approximation around the imaginary axis as provided by Chen in the $g$ method [7]. The quartic formulation of the DLM has been used [3], but note that the modification of the integral terms $I_{0}$ and $J_{0}$ presented above can be used independent of the polynomial order chosen when approximating the required integrand terms in the spanwise direction. 
Chen's approximation [7] uses the Cauchy-Riemann conditions in order to provide a first-order approximation of the complex generalized aerodynamic forces (GAF) matrix $\mathbf{Q}_{h h}$ for the complex variable $p=g+i k$ around the imaginary axis:

$$
\mathbf{Q}_{h h}\left(p, M_{\infty}\right) \approx \mathbf{Q}_{h h}\left(i k, M_{\infty}\right)+g \frac{d \mathbf{Q}_{h h}\left(i k, M_{\infty}\right)}{d(i k)} .
$$

Note that this comparison is valid for small values of the real part of the complex Laplace variable $\operatorname{Re}(p)=g$ because of the inherent limitation to the $g$ method. In contrast to this, the modified DLM proposed here is valid throughout the complex plane.

For the first application the planar AGARD445.6 wing model in its weakened version has been chosen [19]. The resulting GAF matrix $\mathbf{Q}_{h h}\left(p, M_{\infty}\right)$ obtained and projected into the set of the generalized coordinates corresponding to the first four structural modes as provided by Yates [19] is considered:

$$
\mathbf{Q}_{h h}\left(p, M_{\infty}\right)=\boldsymbol{\phi}^{T} \mathbf{H}_{k g}^{T} \mathbf{S}_{k j} \mathbf{D}^{-1}\left(p, M_{\infty}\right)\left(\mathbf{D}_{1 j k}+i \mathbf{D}_{2 j k}\right) \mathbf{H}_{k g} \boldsymbol{\phi} .
$$

In Eq. $12 \mathbf{H}_{k g}$ represents the spline matrix and has been computed applying a thin plate spline (TPS) [20], $\mathbf{S}_{k j}$ is a summation matrix to transfer the pressures to local forces and moments, $\mathbf{D}$ the aerodynamic influence coefficient (AIC) matrix [3], $\mathbf{D}_{1 j k}$ and $\mathbf{D}_{2 j k}$ are the substantial derivative matrices to obtain the downwash caused by the local aerodynamic panel translation and rotation [4] and $\phi$ contains in columns the retained wind-off structural modes.

A representative Mach number $M_{\infty}=0.678$ has been selected. In Figs. 1 to 8 a comparison between the results obtained with the modified DLM and the $g$ method for the diagonal elements of the GAF matrix as function of the reduced frequency $k$ for a constant value of $g=\operatorname{Re}(p)=-0.05$, split into their real and imaginary parts, are shown. For the $g$ method the term $d \mathbf{Q}_{h h}\left(i k, M_{\infty}\right) / d(i k)$ has been approximated by using central finite differences with a spacing $\Delta k=10^{-4}$. Off-diagonal elements of the GAF matrix are not shown but, as for the diagonal elements, GAAM and $g$ formulations cannot be distinguished. Note that the results corresponding to the original DLM formulation of Rodden et al. have been obtained by evaluating their formulation [3] at the transformed variable $p / i=\operatorname{Im}(p)-i \operatorname{Re}(p)$, showing a mismatch with the analytical continuation provided by both the $g$ and GAAM formulations, highlighting the need for the GAAM corrected formulation. 


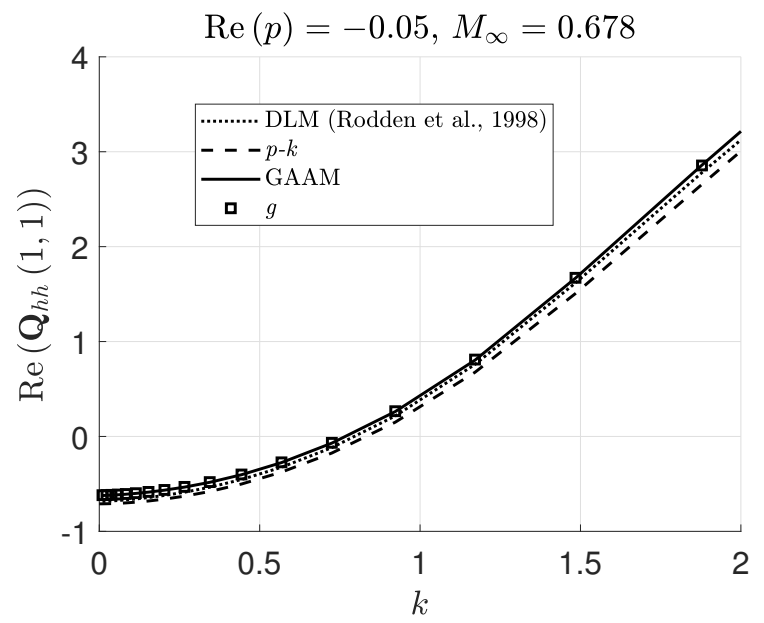

Fig. 1 Real part of component $(1,1)$ of the GAF matrix for $\operatorname{Re}(p)=-0.05$ against the reduced frequency $k$. Mach number $M_{\infty}=0.678$, AGARD445.6 model.

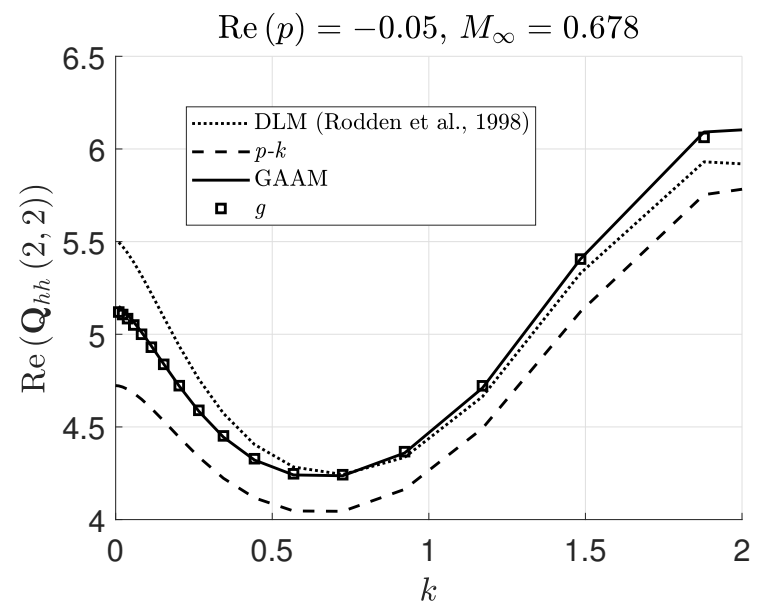

Fig. 3 Real part of component $(2,2)$ of the GAF matrix for $\operatorname{Re}(p)=-0.05$ against the reduced frequency $k$. Mach number $M_{\infty}=0.678$, AGARD445.6 model.

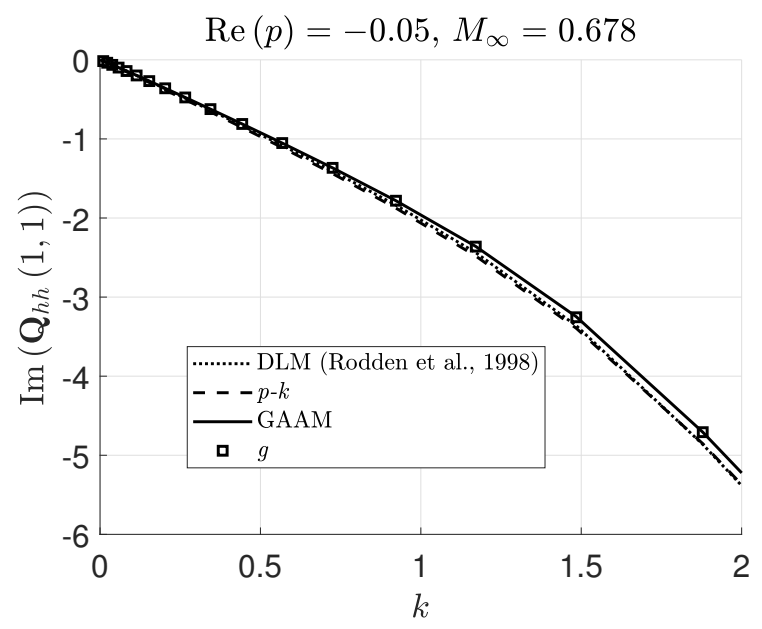

Fig. 2 Imaginary part of component $(1,1)$ of the GAF matrix for $\operatorname{Re}(p)=-0.05$ against the reduced frequency $k$. Mach number $M_{\infty}=0.678$, AGARD445.6 model.

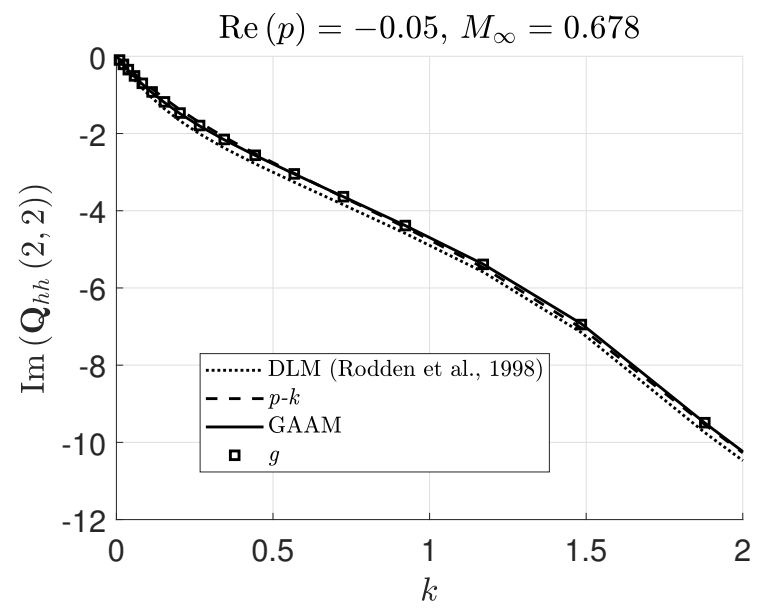

Fig. 4 Imaginary part of component $(2,2)$ of the GAF matrix for $\operatorname{Re}(p)=-0.05$ against the reduced frequency $k$. Mach number $M_{\infty}=0.678$, AGARD445.6 model. 


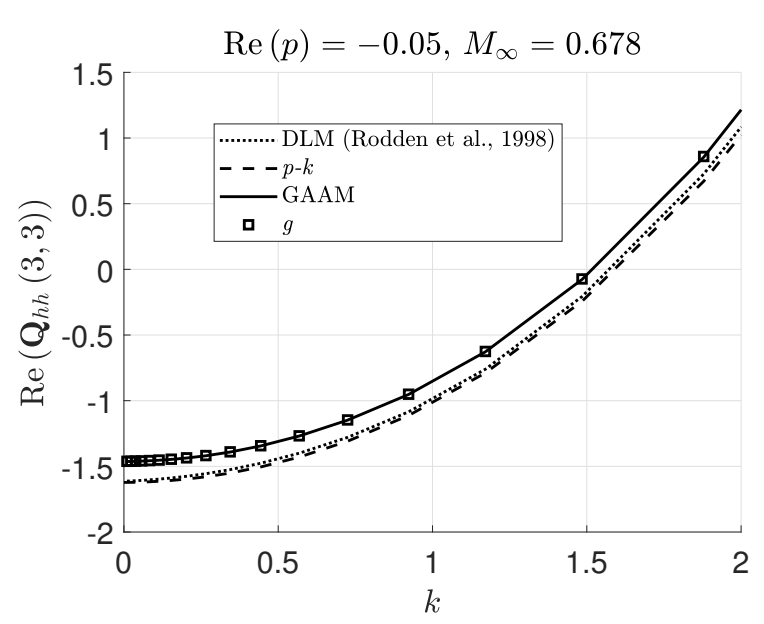

Fig. 5 Real part of component $(3,3)$ of the GAF matrix for $\operatorname{Re}(p)=-0.05$ against the reduced frequency $k$. Mach number $M_{\infty}=0.678$, AGARD445.6 model.

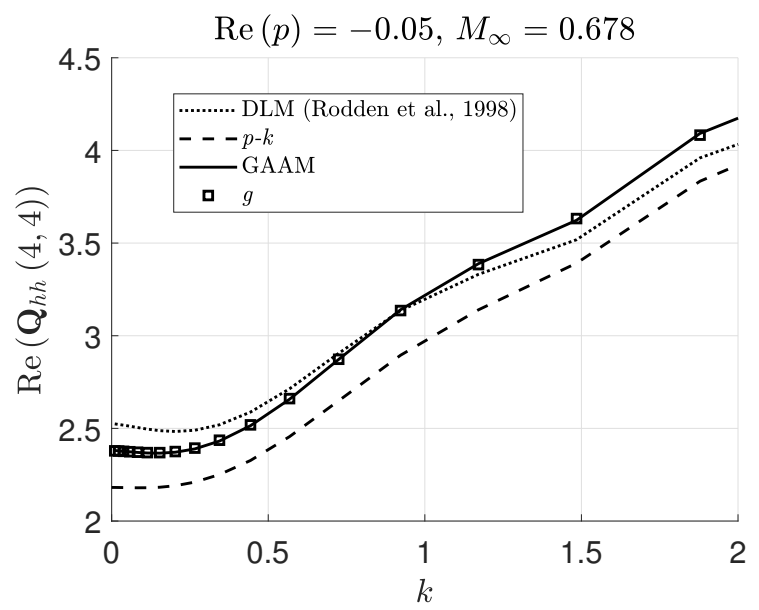

Fig. 7 Real part of component $(4,4)$ of the GAF matrix for $\operatorname{Re}(p)=-0.05$ against the reduced frequency $k$. Mach number $M_{\infty}=0.678$, AGARD445.6 model.

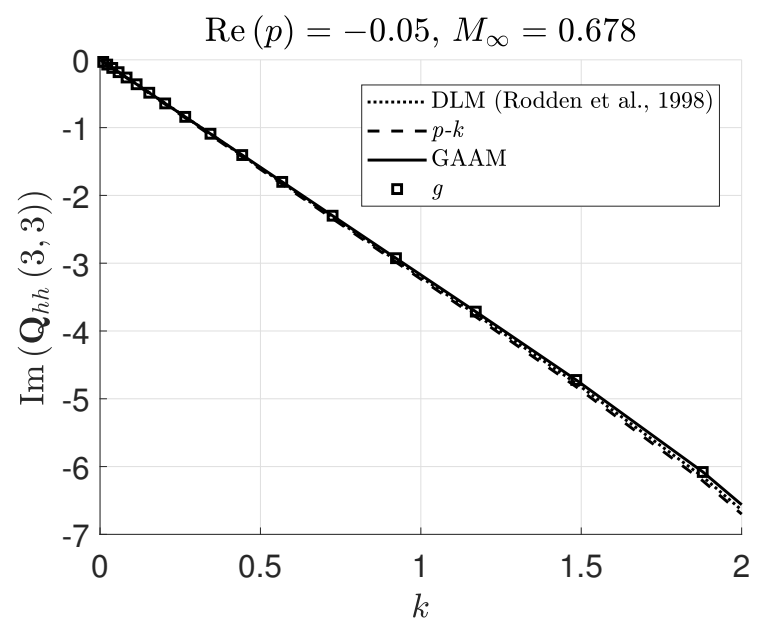

Fig. 6 Imaginary part of component $(3,3)$ of the GAF matrix for $\operatorname{Re}(p)=-0.05$ against the reduced frequency $k$. Mach number $M_{\infty}=0.678$, AGARD445.6 model.

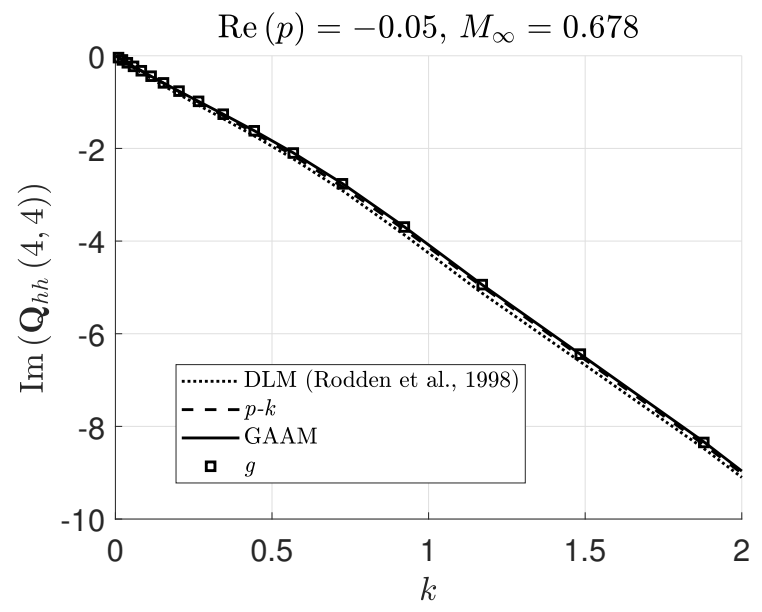

Fig. 8 Imaginary part of component $(4,4)$ of the GAF matrix for $\operatorname{Re}(p)=-0.05$ against the reduced frequency $k$. Mach number $M_{\infty}=0.678$, AGARD445.6 model.

The availability of the GAF matrix as function of the complex variable $p$ eliminates the need for a $p$ - $k$ approximation, as the aerodynamic terms are available throughout the complex plane. Thus, a flutter solver [21] can be readily adapted to consider the additional dependency of the GAF matrix on the complex Laplace variable $p$, so that the aeroelastic roots are properly computed, representing an actual implementation of GAAM as proposed by Edwards and Wieseman [14] for general three-dimensional nonplanar configurations. Compared with the $g$ method, which predicts the aeroelastic 
roots away from the flutter point provided their real part are small, the GAAM proposed here is valid for excursions of the aeroelastic roots further away from the imaginary axis. When computing the aeroelastic roots as the solution to a nonlinear eigenvalue problem [21] and because of the GAF availability at a number of precomputed $p$ values, this matrix is interpolated linearly with respect to the real and imaginary parts of the complex variable $p$.

Fig. 9 shows the aeroelastic damping (scaled as $2 g / k$ ) as predicted by the $p-k$ and $g$ methods and GAAM against the speed index $F_{i}=U_{\infty} /\left(L_{r e f} \omega_{\theta} \sqrt{\mu}\right)$, where $U_{\infty}$ is the freestream velocity, $L_{r e f}=0.5578(\mathrm{~m})$ a reference length, $\omega_{\theta}=38.165(\mathrm{~Hz})$ the torsional frequency and $\mu=68.753$ the mass ratio. The corresponding density is $\rho_{\infty}=0.2082$ $\left(\mathrm{kg} / \mathrm{m}^{3}\right)$. For this particular aeroelastic model and for the range of parameters selected, the $g$ method and GAAM provide equivalent results. For increasing damping, that is, real part of the aeroelastic roots, the $g$ method will eventually deviate from the true GAAM prediction. The flutter condition is given at $F_{i F}=0.429$, close to the experimental value of $F_{i F}=0.416[19]$.

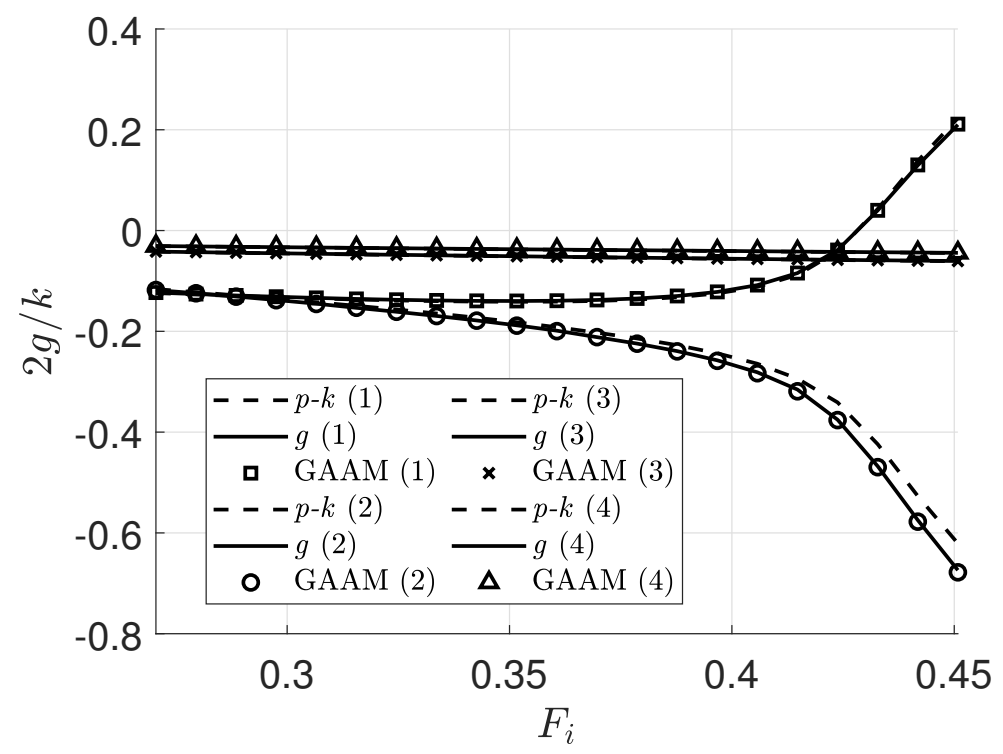

Fig. 9 Damping $(2 g / k)$ at $M_{\infty}=0.678$ and $\rho_{\infty}=0.2082\left(\mathrm{~kg} / \mathrm{m}^{3}\right)$ against speed index $F_{i}$. AGARD 445.6 model.

In order to show the validity of the modified DLM implementation for a nonplanar geometry, a long-range transport aircraft configuration corresponding to the NASA common research model (CRM) geometry [22] as created by Klimmek [23] has been considered next. The $\mathrm{C} 2$ case has been chosen, corresponding to a mass equal to the maximum takeoff weight of $260000(\mathrm{~kg})$. The geometry is in the deformed flight shape and presents a dihedral angle for the wing and the horizontal tail plane components with a total of 2150 aerodynamic panels resulting in a nonplanar configuration, for which the integral $I_{2}$ is non-zero, requiring the computation of both $I_{0}$ and $J_{0}$ integrals. A more detailed description of the structural and aerodynamic models is provided by Klimmek [23]. The first 44 structural modes together with 6 rigid-body modes for the full-model configuration have been considered in the matrix $\phi$. For the sake of brevity Figs. 10 to 13 show the results in real and imaginary parts corresponding to the combinations of the aircraft heave 
and pitch rigid-body motion for a constant value of $g=\operatorname{Re}(p)=-0.02$, but equivalent conclusions can be drawn for other components of the GAF matrix. As for the planar configurations, the proposed GAAM formulation and the $g$ method cannot be distinguished. As for the previous model, the results corresponding to the original DLM formulation of Rodden et al. [3] have been obtained by evaluating their formulation [3] at the transformed variable $p / i=\operatorname{Im}(p)-i \operatorname{Re}(p)$. Figs. 16 and 17 show the results for the pitch/pitch GAF component at a higher damping value of $g=\operatorname{Re}(p)=-0.06$, whereas Figs. 14 and 15 depict the GAF component corresponding to the first flexible mode for a value of $g=\operatorname{Re}(p)=0.2$. It can be observed that for an increasing magnitude of the value $|g|=|\operatorname{Re}(p)|$, the $g$ method will depart from the reference GAAM presented here, which can now be used as a validation tool for the $g$ method when DLM aerodynamics is used.

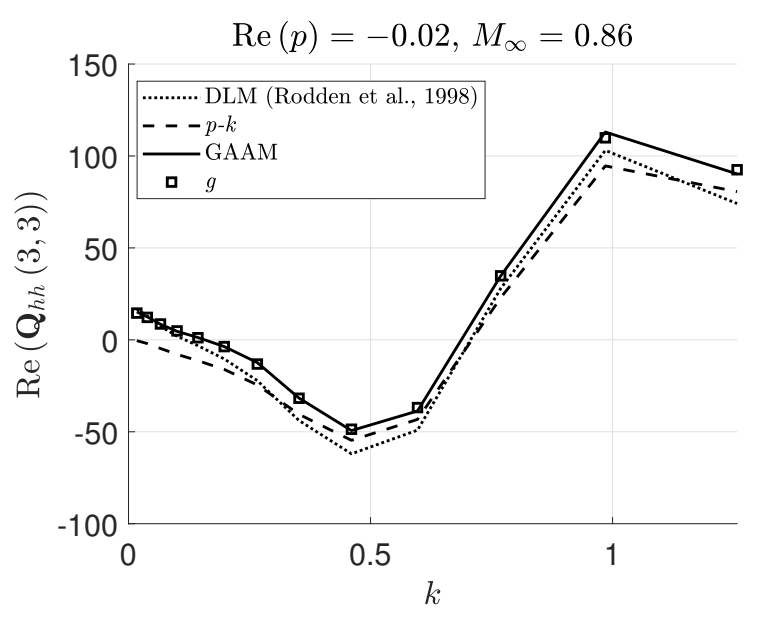

Fig. 10 Real part of heave/heave component $(3,3)$ of the GAF matrix for $\operatorname{Re}(p)=-0.02$ against the reduced frequency $k$. Mach number $M_{\infty}=0.86$, CRM.

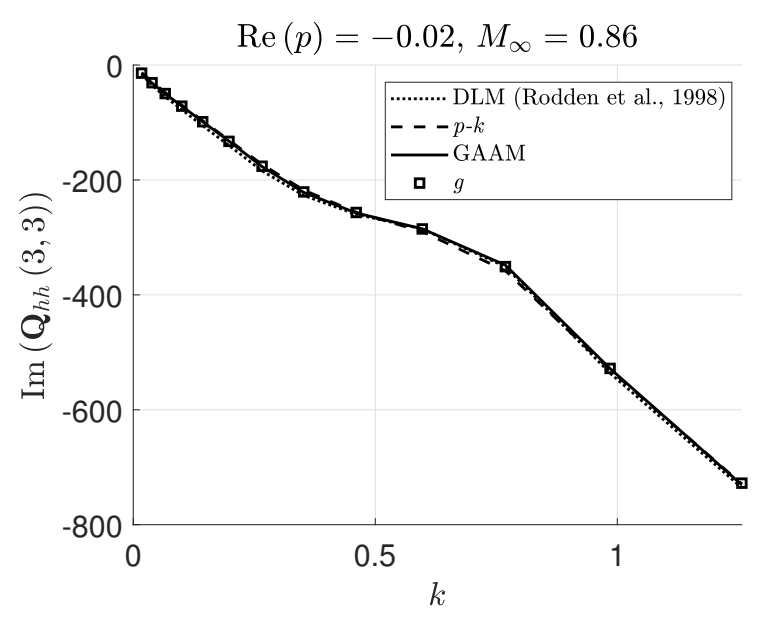

Fig. 11 Imaginary part of heave/heave component $(3,3)$ of the GAF matrix for $\boldsymbol{R e}(p)=-0.02$ against the reduced frequency $k$. Mach number $M_{\infty}=0.86$, CRM. 


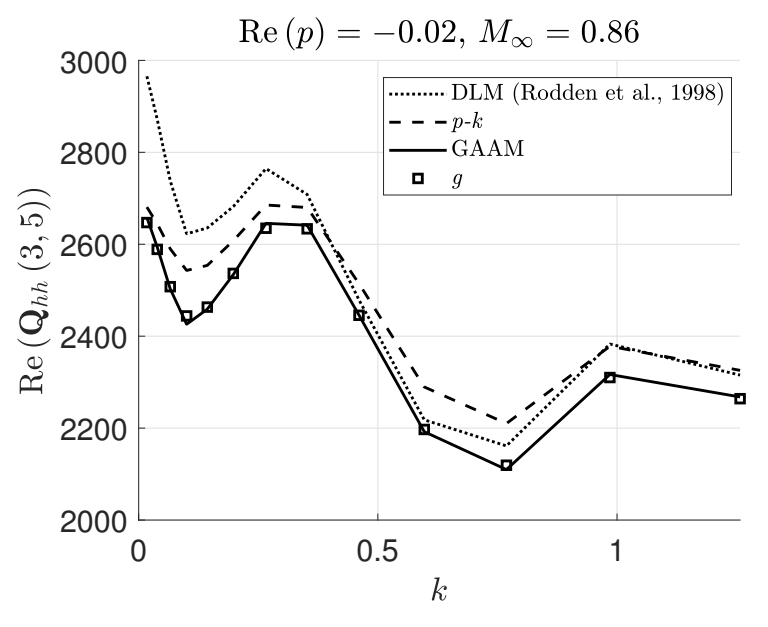

Fig. 12 Real part of heave/pitch component $(3,5)$ of the GAF matrix for $\operatorname{Re}(p)=-0.02$ against the reduced frequency $k$. Mach number $M_{\infty}=0.86$, CRM.

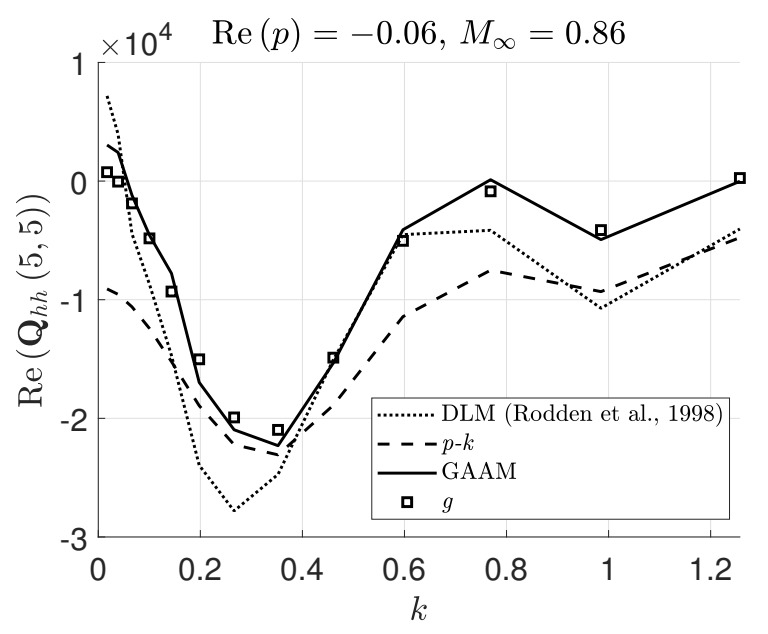

Fig. 14 Real part of pitch/pitch component $(5,5)$ of the GAF matrix for $\operatorname{Re}(p)=-0.06$ against the reduced frequency $k$. Mach number $M_{\infty}=0.86$, CRM.

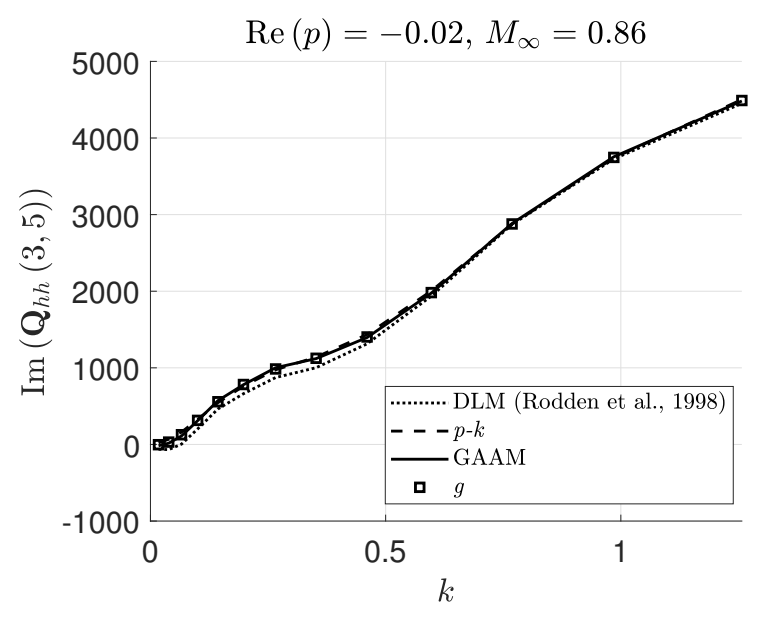

Fig. 13 Imaginary part of heave/pitch component $(3,5)$ of the GAF matrix for $\operatorname{Re}(p)=-0.02$ against the reduced frequency $k$. Mach number $M_{\infty}=$ 0.86, CRM.

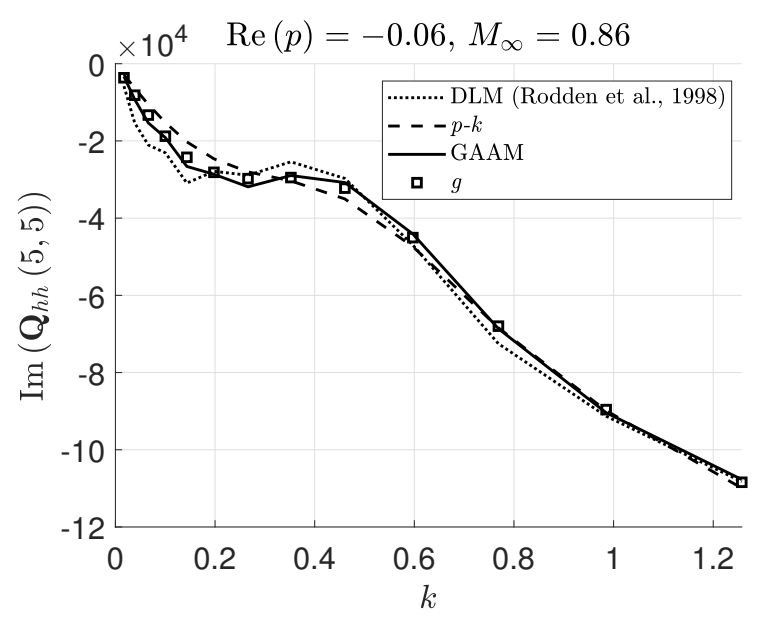

Fig. 15 Imaginary part of pitch/pitch component $(5,5)$ of the GAF matrix for $\operatorname{Re}(p)=-0.06$ against the reduced frequency $k$. Mach number $M_{\infty}=$ 0.86, CRM. 


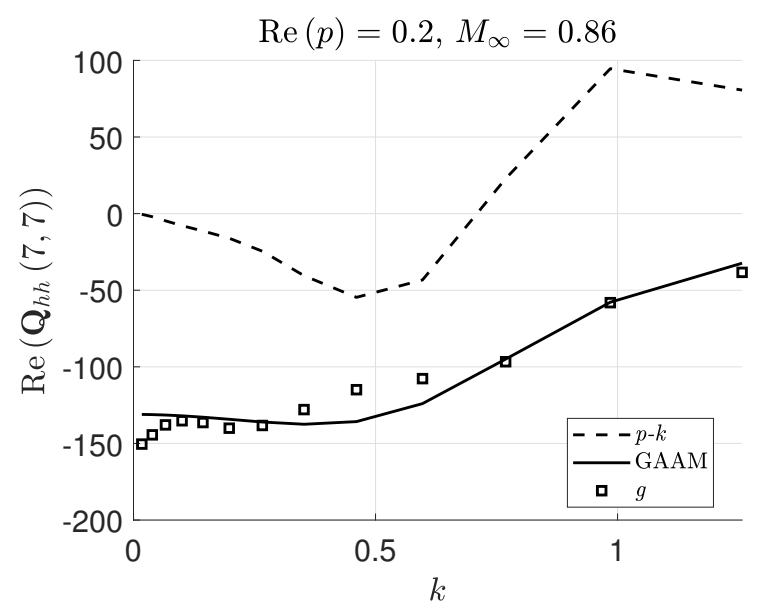

Fig. 16 Real part of flexible/flexible component $(7,7)$ of the GAF matrix for $\operatorname{Re}(p)=0.2$ against the reduced frequency $k$. Mach number $M_{\infty}=$ 0.86 , CRM.

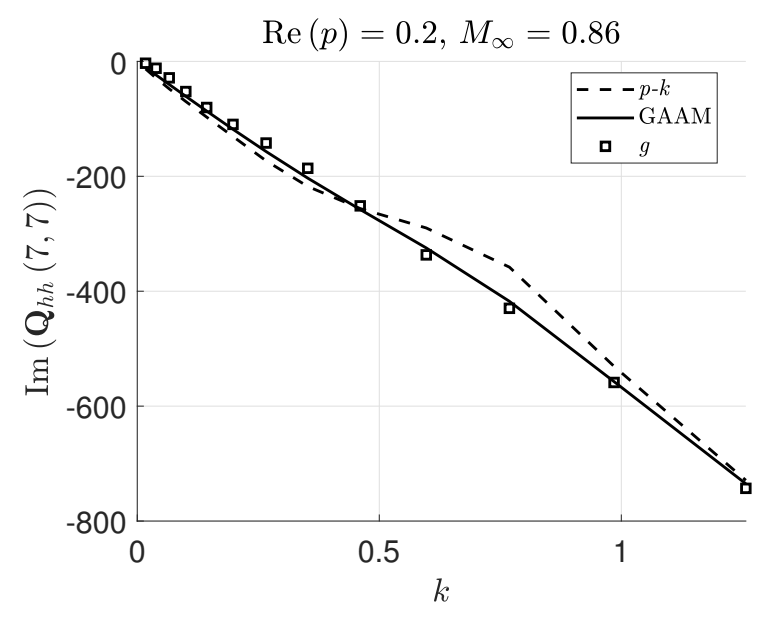

Fig. 17 Imaginary part of flexible/flexible component $(5,5)$ of the GAF matrix for $\operatorname{Re}(p)=0.2$ against the reduced frequency $k$. Mach number $M_{\infty}=0.86$, CRM.

Fig. 18 shows the evolution of the scaled damping $2 g / k$ of the aeroelastic modes at the same Mach number against the true airspeed $U_{\infty}$ for a fixed value of the density $\rho_{\infty}=1.225\left(\mathrm{~kg} / \mathrm{m}^{3}\right)$ as obtained by considering the first 44 flexible modes. In order to highlight the difference between the $g$ method and GAAM, the CRM has been weakened by multiplying the generalized stiffness matrix by a factor of $1 / 2$ while keeping the same eigenvectors in wind-off conditions and leaving the generalized mass equal to the identity matrix. For the sake of clarity, only the modes which become unstable (12 and 13 correspond to the $6^{\text {th }}$ and $7^{\text {th }}$ flexible modes) together with those presenting a higher damping value in magnitude have been depicted. As expected from the results shown in Figs. 10 to 15 , the evolution of the damping for the $g$ method and GAAM are indistinguishable except for higher damping values occurring for the modes 12 and 13 at high speeds. Still, the $g$ method provides valuable results for these two particular configurations in a wide range of velocities. 


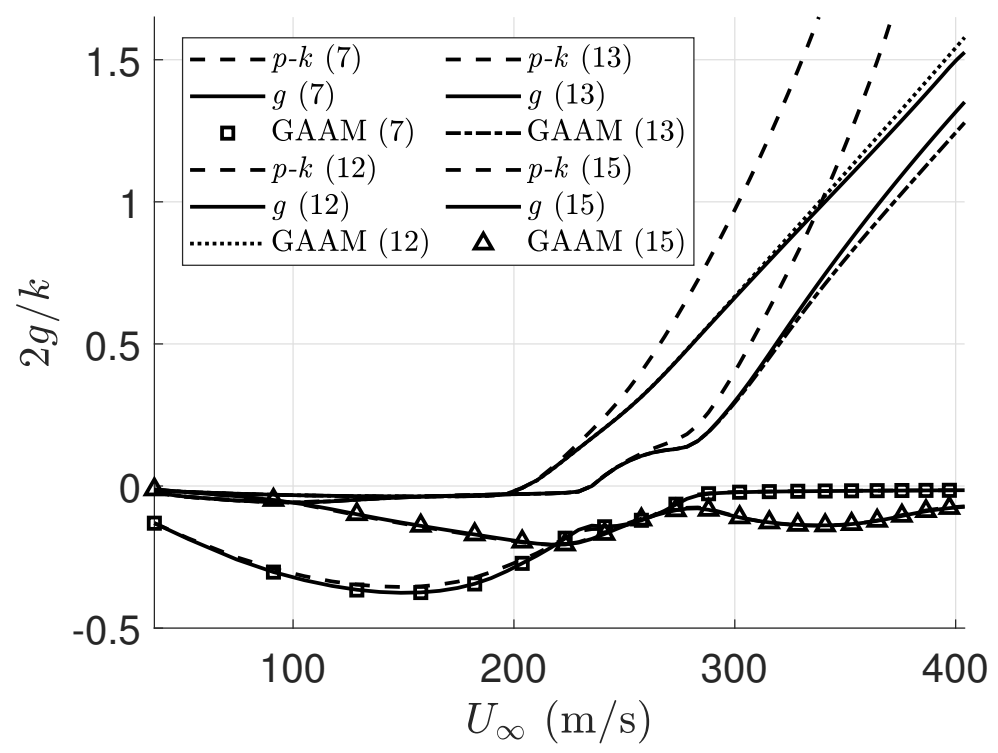

Fig. 18 Damping $(2 g / k)$ at $M_{\infty}=0.86$ and $\rho_{\infty}=1.225\left(\mathbf{k g} / \mathbf{m}^{3}\right)$ against true airspeed $U_{\infty}(\mathbf{m} / \mathbf{s})$. Weakened CRM.

In order to further highlight the potential difference between existing methods and the GAAM at lower speeds in the stable region, another modification of the CRM has been considered at a generalized level by multiplying the generalized mass and stiffness matrices by a factor of $1 / 2$ and 3/2 respectively. Again, for small absolute values of $g$ the $p-k$ and $g$ methods can be used but for increasing absolute values the $p-k$ method first and later the $g$ method eventually differ from the reference GAAM, as shown for the mode 7 in Fig. 19 Interestingly, the value of $g$ at which the $p-k$ and $g$ methods differ from the reference provided by the GAAM depends on the airspeed $U_{\infty}$. As for the previous cases, the three methods predict the same airspeed for the instability $(g=0)$, shown by the mode 8 . 


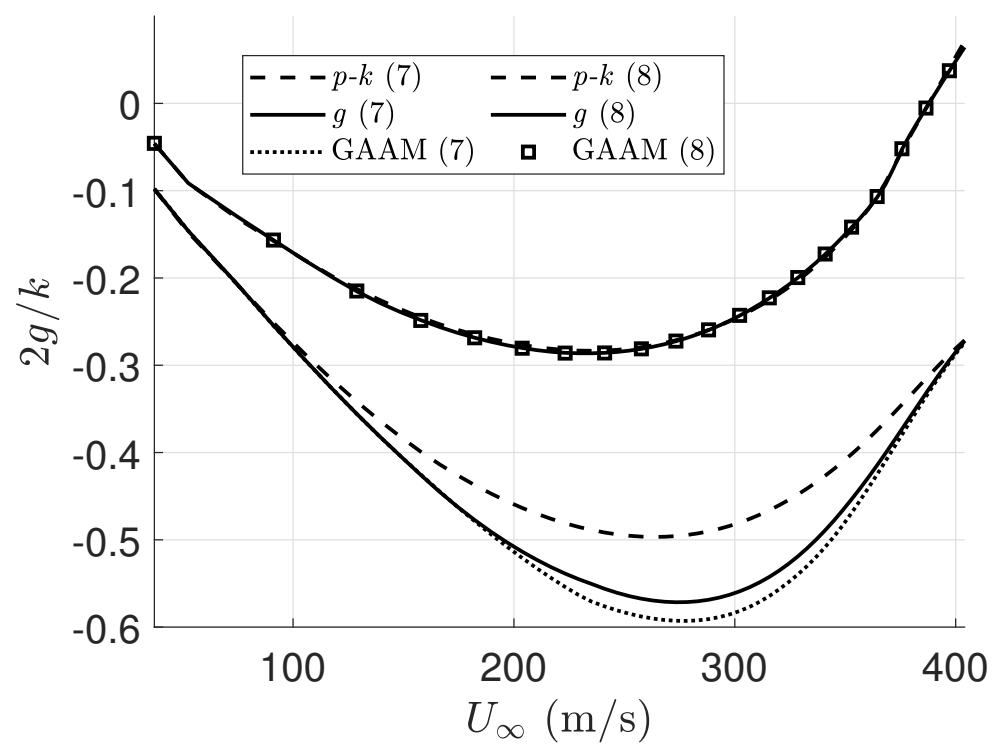

Fig. 19 Damping $(2 g / k)$ at $M_{\infty}=0.86$ and $\rho_{\infty}=1.225\left(\mathbf{k g} / \mathbf{m}^{3}\right)$ against true airspeed $U_{\infty}(\mathbf{m} / \mathbf{s})$. Modified CRM.

\section{Conclusions}

In this note a modified formulation of the doublet lattice method applicable throughout the complex plane has been presented and verified. This formulation allows for a direct application of the generalized aeroelastic analysis method for general nonplanar configurations in subsonic flow, providing an alternative method with an extended range of validity for configurations with high aeroelastic damping and a means of determining the suitability of the $g$ method.

\section{References}

[1] Albano, E., and Rodden, W., "A doublet-lattice method for calculating lift distributions on oscillating surfaces in subsonic flows," AIAA Journal, Vol. 7, No. 2, 1969, pp. 279-285. https://doi.org/10.2514/3.5086

[2] Wright, J., and Cooper, J., Introduction to aircraft aeroelasticity and loads, Vol. 20, John Wiley \& Sons, 2008. //doi.org/10.1002/9781118700440

[3] Rodden, W. P., Taylor, P. F., and McIntosh, S. C., "Further Refinement of the Subsonic Doublet-Lattice Method," Journal of Aircraft, Vol. 35, No. 5, 1998, pp. 720-727. https://doi.org/10.2514/2.2382

[4] Rodden, W., Harder, R., and Bellinger, E., “Aeroelastic addition to NASTRAN,” NASA Contractor Report $3094,1979$.

[5] Rodden, W., and Johnson, E., MSC/NASTRAN Aeroelastic Analysis User's Guide, Version 68, MacNeal-Schwendler Corporation, 1994.

[6] Hassig, H., "An approximate true damping solution of the flutter equation by determinant iteration," Journal of Aircraft, Vol. 8, No. 11, 1971, pp. 885-889. https://doi.org/10.2514/3.44311. 
[7] Chen, P., "Damping Perturbation Method for Flutter Solution: The g-Method," AIAA Journal, Vol. 38, No. 9, 2000 , pp. 1519-1524. https://doi.org/10.2514/2.1171.

[8] Edwards, J., "Unsteady aerodynamic modeling and active aeroelastic control,” Ph.D. thesis, Stanford University, 1977.

[9] Edwards, J., "Unsteady Aerodynamic Modeling for Arbitrary Motions," AIAA Journal, Vol. 15, No. 4, 1977, pp. $593-595$. https://doi.org/10.2514/3.60664

[10] Edwards, J., Ashley, H., and Breakwell, J., “Unsteady aerodynamic modeling for arbitrary motions," AIAA Journal, Vol. 17, No. 4, 1979, pp. 365-374. https://doi.org/10.2514/3.7348

[11] Edwards, J., "Applications of Laplace transform methods to airfoil motion and stability calculations," 20th Structures, Structural Dynamics, and Materials Conference, St. Louis, MO, USA, 1979. https://doi.org/10.2514/6.1979-772.

[12] Cunningham, H., and Desmarais, R., "Generalization of the subsonic kernel function in the s-plane, with applications to flutter analysis," NASA Technical Paper 2292, 1984.

[13] Hounjet, M., and Eussen, B., "Beyond the frequency limits of time-linearized methods," International Forum on Aeroelasticity and Structural Dynamics (IFASD), Aachen, Germany, 1991.

[14] Edwards, J., and Wieseman, C., "Flutter and Divergence Analysis Using the Generalized Aeroelastic Analysis Method," Journal of Aircraft, Vol. 45, No. 3, 2008, pp. 906-915. https://doi.org/10.2514/1.30078

[15] Giesing, J., Kalman, T., and Rodden, W., "Subsonic unsteady aerodynamics for general configurations. Part I, Vol I - Direct application of the nonplanar doublet-lattice method," Technical Report AFFDL-TF-71-5, 1971, p. 26.

[16] Watkins, C. E., Woolston, D. S., and Cunningham, H. J., "A systematic kernel function procedure for determining aerodynamic forces on oscillating or steady finite wings at subsonic speeds," NASA Technical Report R-48, 1959.

[17] Laschka, B., "Zur Theorie der harmonisch schwingenden tragenden Fläche bei Unterschallanströmung," Zeitschrift für Flugwissenschaften, Vol. 11, No. 7, 1963, pp. 265-292.

[18] Desmarais, R., "An accurate and efficient method for evaluating the kernel of the integral equation relating pressure to normalwash in unsteady potential flow," 23rd Structures, Structural Dynamics and Materials Conference, New Orleans, LA, USA, 1982. https://doi.org/10.2514/6.1982-687.

[19] Yates, E., "AGARD standard aeroelastic configurations for dynamic response. Candidate configuration I.-wing 445.6," AGARD Report No.765, 1987.

[20] Duchon, J., "Splines minimizing rotation-invariant semi-norms in Sobolev spaces," Constructive Theory of Functions of Several Variables, edited by W. Schempp and K. Zeller, Springer Berlin Heidelberg, 1977, pp. 85-100. https://doi.org/10.1007/ $\mathrm{BFb0086566}$ 
[21] Cardani, C., and Mantegazza, P., "Continuation and direct solution of the flutter equation,” Computers and Structures, Vol. 8, No. 2, 1978, pp. 185 - 192. https://doi.org/10.1016/0045-7949(78)90021-4

[22] Vassberg, J., Dehaan, M., Rivers, M., and Wahls, R., "Development of a common research model for applied CFD validation studies," 26th AIAA applied aerodynamics conference, Honolulu, Hawaii, USA, 2008, p. 6919. https://doi.org/10.2514/6.20086919

[23] Klimmek, T., "Parametric set-up of a structural model for FERMAT configuration aeroelastic and loads analysis," Journal of Aeroelasticity and Structural Dynamics, Vol. 3, No. 2, 2014. https://doi.org/10.3293/asdj.2014.27 